Revue européenne des sciences sociales

European Journal of Social Sciences

XLVI-140 2008

La sociologie en quête d'une théorie générale

\title{
La science sociale de Vilfredo Pareto
}

\section{Giovanni Busino}

\section{OpenEdition}

Journals

Édition électronique

URL : http://journals.openedition.org/ress/172

DOI : $10.4000 /$ ress. 172

ISSN : 1663-4446

\section{Éditeur}

Librairie Droz

\section{Édition imprimée}

Date de publication : 1 février 2008

Pagination : 107-132

ISBN : 978-2-600-01221-8

ISSN : 0048-8046

Référence électronique

Giovanni Busino, "La science sociale de Vilfredo Pareto », Revue européenne des sciences sociales [En ligne], XLVI-140 | 2008, mis en ligne le 01 février 2011, consulté le 10 décembre 2020. URL : http:// journals.openedition.org/ress/172 ; DOI : https://doi.org/10.4000/ress.172 


\section{Giovanni BUSINO}

\section{LA SCIENCE SOCIALE DE VILFREDO PARETO}

\section{PROLOGUE}

Pareto est sans doute, parmi tous les sociologues classiques, celui qui a été et reste le plus contesté en histoire de la sociologie. Les critiques de son œuvre ont été et sont nombreuses, disparates, et certaines d'entre elles conditionnent encore la réception et la compréhension de théories parétiennes chez les sociologues contemporains (Busino, 1974; 1999: 23-33; 2006: 660-680; 2007: 127-385). Aux chercheurs d'aujourd'hui, en effet, les problématiques élaborées par Pareto paraissent irritantes, insupportables, impertinentes (Freund, 1974: 5-22), voire caduques ou obsolètes, malgré ses tentatives de faire de la recherche sociale la synthèse de toutes les sciences humaines, d'englober l'économie dans la sociologie et l'histoire (Pareto, 1987: 146-161 ; Passeron, 1995 : 37-137; 2000: 25-71), d'élaborer un modèle d'homme social, «raisonneur et raisonnant», de décrire les variations des usages du principe de rationalité par les acteurs historiques, de «construire» une science logico-expérimentale des contextes historiques caractérisés par la «dépendance mutuelle des conditions».

Il est vrai que la sociologie parétienne délaisse les méthodes et les procédures standard de la recherche, qu'elle méconnaît les structures normatives et morales des faits sociaux et leurs implications (normatives et non normatives) d'ordre moral et politique. Mais puisque Pareto admet l'existence de fins (l'autoconservation et la reproduction) dans l'ordre naturel du vivant, la détermination des actions sociales par des prédispositions de comportements sélectionnés phylogénétiquement, la possibilité d'inférer les actions et les représentations manifestes des éléments interdépendants constitutifs de ces prédispositions «naturelles », il paraît charitable et profitable de reconnaître que ces problématiques méritent d'être analysées et critiquées de façon attentive et scrupuleuse d'autant plus qu'elles n'ont pas jusqu'ici été toutes éclaircies ou déconstruites par la recherche sociologique et par l'épistémologie sociale contemporaines.

\section{UNE «SOCIOLOGIE EXCLUSIVEMENT EXPÉRIMENTALE»}

«Nous sommes riches de sociologies humanitaires, comme presque toutes celles qui se publient maintenant. Nous ne manquons pas de sociologies métaphysiques et parmi elles, il faut ranger toutes les positivistes et toutes les humanitaires. Nous avons un certain nombre de sociologies chrétiennes, catholiques ou 
autres. Qu'on nous permette, sans vouloir faire tort à toutes ces estimables sociologies, d'en exposer ici une exclusivement expérimentale comme la chimie, la physique et d'autres sciences du même genre» (Pareto, 1968: § 6).

Qu'est-ce que c'est cette «sociologie exclusivement expérimentale» que Pareto, après avoir abandonné les études d'économie pure, veut élaborer?

La mise en forme d'un modèle pour l'étude des mécanismes économiques lui avait révélé les avantages de la méthode des approximations successives, mais ses limites aussi. Expliquer, comprendre et surtout prévoir sont des activités incertaines, indéterminablés, peu sûres, de toute façon longues et périlleuses. Les résultats ne sont ni immédiats ni univoques car la recherche scientifique procède au pas, par itérations qui se succèdent indéfiniment. La science économique, construction abstraite, langue artificielle, aide à réaliser une première approximation vers la compréhension des conduites humaines finalisées et vers les conditions par lesquelles ces conduites se composent ou s'opposent. Cette première approximation est rudimentaire, partielle. Pour réaliser d'autres approximations l'économie pure doit s'ouvrir à l'économie appliquée et puis à la sociologie et à l'histoire.

Derrière la cautèle des approximations successives, de la science comme tâtonnement et rectification perpétuelle, il y a une métathéorie, voire un présupposé, clairement énoncé dès les débuts, selon lequel la science est recherche approximative et réductrice d'une réalité complexe, qui ne sera jamais reconstruite en sa totalité ou délinée intégralement. Néanmoins, seules ces réalités partielles ou relatives peuvent nous aider à voir clair dans nos comportements. Elles seules, en présumant la combinaison des moyens en vue d'une fin, nous offrent les instruments afin de rationaliser éventuellement nos conduites.

La science n'étant ni une reconstruction-restitution de la société, ni un pur reflet de celle-ci, ni une copie plus ou moins impressionniste du réel, mais bien une activité par laquelle ou en vertu de laquelle l'homme essaie d'expliquer les raisons de son propre agir ou les connexions des événements, Pareto doit élaborer un langage abstrait grâce auquel il peut ensuite construire des objets scientifiques.

L'activité scientifique est donc l'édification d'un univers simplifié, extrait artificiellement de l'univers concret, sur lequel le savant doit travailler. La science n'épuise jamais la richesse et la variété du réel, l'hétérogénéité des faits sociaux. La construction de ces objets scientifiques est possible grâce à des opérations abstractives (les modèles et les théories), lesquelles corrigent les représentations soumises aux influences des croyances, des idéologies, des intérêts, des aspirations individuelles et collectives. C'est la théorie (des concepts abstraits plus ou moins organisés) qui fabrique les faits sociaux et leur donne de la consistance et de la cohérence, c'est la théorie qui établit les liens structurant les évènements, les relations entre les phénomènes, c'est elle, en bref, qui construit l'objet scientifique.

L'intelligibilité n'est pas une propriété du réel, elle est le résultat des reconstructions scientifiques, qu'il faut toujours comparer entre elles afin de réduire les effets de la subjectivité du savant. Pour cette raison il faut élaborer des modèles pour chaque activité sociale et percevoir celle-ci sous l'angle de ses relations fondamentales. Claude Lévi-Strauss a dit presque la même chose à propos d'une catégorie particulière de ces objets: "A la suite de Rousseau, et sous une forme décisive, Marx a enseigné que la science sociale ne se bâtit pas plus sur le plan des 
événements que la physique à partir des données de la sensibilité: le but est de construire un modèle, d'étudier ses propriétés et les différentes manières dont il réagit au laboratoire, pour appliquer ensuite ces observations à l'interprétation de ce qui se passe empiriquement» (Lévi-Strauss,1966: 44). Et dans cette même perspective les auteurs de Le métier de sociologue ont glosé: "Ainsi c'est à son pouvoir de rupture et à son pouvoir de généralisation, les deux étant inséparables, que l'on reconnaît le modèle théorique: épure formelle des relations entre les relations qui définissent les objets construits, il peut être transposé à des ordres de réalité phénoménalement très différents et suggérer par analogie des nouvelles analogies, principes de nouvelles constructions d'objets» (Bourdieu, Chamboredon, Passeron, 1973: 79).

La conception de la théorie de Pareto est, certes, aussi ambiguë que celles en vogue dans les sciences sociales d'aujourd'hui (Boudon, 1971: 159-204). Toutefois relisons-la dans sa formulation la plus succincte: "On trouve, dans une théorie, des parties descriptives, des affirmations axiomatiques, l'intervention d'êtres concrets ou abstraits, réels ou imaginaires. Tout cela constitue en quelque sorte les matériaux de cette théorie. On y trouve aussi des raisonnements logiques ou pseudo- logiques, un appel aux sentiments, des développements pathétiques, l'intervention d'éléments éthiques, religieux, etc. Tout cela donne en somme la façon dont on met en œuvre les matériaux, pour construire l'édifice qu'on appelle une théorie» (Pareto, 1968: § 12).

Une telle conception nominaliste de la science fonde et conditionne toute la recherche parétienne, sa méthode logico-expérimentale grâce à laquelle l'auteur imagine et forge les variables abstraites, établit les interdépendances, obtient les «vérités expérimentales ». "Nous nous mouvons - écrit Pareto -, dans un champ restreint; dans celui de l'expérience et de l'observation, sans nier qu'il y en ait d'autres, mais avec la volonté bien arrêtée de ne pas nous en occuper ici. Notre but est de découvrir des théories qui représentent les faits de l'expérience et de l'observation [...] car loin de nier l'utilité sociale d'autres théories, nous croyons même qu'elles peuvent être très profitables». Toutefois «associer l'utilité sociale d'une théorie à sa vérité expérimentale est justement un de ces principes à priori que nous repoussons» (Pareto, 1968: $\S \S 71-72$ ).

L'expérience et l'observation en tant que telles ont-elles donc des fondements ontologiques? Si la première est une action et une construction progressive, une structuration graduelle de nos opérations par la voie d'assimilation et d'accommodation; si la seconde consiste à considérer les choses telles qu'elles sont, à en découvrir les propriétés, à les coordonner, à les reproduire mentalement, alors comment faire abstraction des conditionnements sociaux, du particularisme des points de vue, des différences culturelles et de classe, en bref du fait que ni l'expérience ni l'observation ne sont adiaphores et qu'elles ne fournissent jamais des images eidétiques?

Ces questions sur le caractère social, changeant, variable, arbitraire de nos manières de penser et d'agir ne sont pas directement traitées. Pour Pareto il y a un dualisme radical entre les propositions cognitives et celles ayant des fonctions normatives et de prescriptions, entre les faits et les valeurs, entre ce qui est et ce qui doit être. Les faits ne sont pas soumis aux lois et par ailleurs celles-ci ne sont pas nécessaires. Les lois sont des «hypothèses qui servent à résumer un nombre plus ou moins grand de faits, et durent tant qu'on ne leur en substitue pas de 
meilleures. Toutes les recherches sont contingentes, relatives, et donnent des résultats qui ne sont que plus ou moins probables, tout au plus très probables. [...] Toutes nos propositions, y compris celles de pure logique, doivent être entendu avec la restriction: dans les limites du temps et de l'expérience à nous connus.» (Pareto, 1968: $\S 69$ et 97).

Pareto accepte sans perplexité les produits de l'expérience et de l'observation, il est très attentif aux significations émotionnelles des mots, aux affects et passions suscités par le vocabulaire mais il répète que les mots du langage descriptif restent amphibologiques et polysémiques (Pareto, 1968: § 442), que l'auto-observation, la mise à plat des discours rationalisant les sentiments, l'analyse des langages ordinaires permettent de décrire ou d'expliquer la régularité des conduites sociales ainsi que le sens culturel des uniformités sociales des acteurs historiquement identifiés.

\section{LA CONSTRUCTION DE L'OBJET SCIENTIFIQUE}

$\mathrm{Au}$ commencement de toute chose, il y a l'action; l'ensemble des actions constitue la trame de ce qu'on appelle communément la société humaine. L'action est un comportement orienté vers des objectifs, elle est un développement unique à travers des situations multiples, elle est un effort, une dépense d'énergies impliquant au moins un motif ou un mobile; elle est toujours chargée de multiples significations L'action est l'unité de base de toute recherche. Le savant doit analyser les actions du dehors, comme si elles étaient détachées «des sujets conscients qui se les représentent» (Pareto, 1968: $\S \S 22,490,582)$. Il « construit» les faits, il les interprète en «constituant» des causalités, des finalités, des correspondances, des subordinations, en bref des relations diverses. Cette multiplicité de causes diverses agissant corrélativement, lui permet de valoriser les « rapports de mutuelle dépendance, qui donnent naissance à une suite d'actions et de réactions » (Pareto, 1968: $§ 1731$ ), de soutenir que l'action a une nature rythmique ou oscillatoire et que ses formes passent à travers différentes phases, lesquelles recommencent toujours dans un même ordre. Le développement social étant déterminé par un grand nombre de conditions s'impliquant réciproquement, «les faits sociaux, c'est-à-dire conditions et effets, sont mutuellement dépendants; une modification de l'un se répercute sur une partie plus ou moins grande des autres, avec une intensité plus ou moins forte.»(Pareto, 1968: $§ 138$ ). Bien entendu, le fait expérimental ne couvre pas la totalité du phénomène concret. Le processus d'abstraction de la science est analytique et pas expérimental.

Comment décrire un «fait social» si complexe dans toutes ses interdépendances? Il faut, préalablement, élaborer une théorie à l'aide de laquelle il est possible de construire et puis de décrire l'action comme étant un objet-système. Cette approche n'est pas dissemblable de celle de certains structuralistes qui déduisent de la théorie les propriétés de l'objet-système. (Boudon, 1968: 79-85). L'exemple des recherches sur la distribution des revenus dans les sociétés d'hier et d'aujourd'hui est vraiment paradigmatique (Pareto, 1964: II, 299-345; 1965 : 43-48).

Pareto essaie de comprendre comment et pourquoi la richesse, ou plus précisément les revenus se répartissent-ils parmi les divers groupes sociaux de sociétés 
différentes. L'observation suggère qu'au delà de différences particulières et des contingences historiques, la répartition n'est pas gouvernée par le hasard ou par des accidents aléatoires propres à tel ou tel groupe social et société humaine. Une théorie appropriée unit les nombreuses observations empiriques (essentiellement les statistiques fiscales) et déduit ensuite les caractéristiques profondes de l'objetsystème. En d'autres termes, la théorie donne de l'unité à des matériaux disparates, rend compte de la stratification économique et de là tire par déduction le pourquoi de tel phénomène. Dans ce cas de la distribution ou répartition des revenus, Pareto constate que, dans tout société, à toute époque, le phénomène prend la forme d'une pyramide ou d'une toupie renversée, et cela indépendamment des conditions économiques et sociales les plus diverses. La théorie présuppose que cette forme (soit la forme rigide de la pyramide soit la forme flexible de la toupie renversée) ne dépend pas du hasard; si, par contre, il en était ainsi, la forme devrait être semblable à la courbe des probabilités ou des erreurs. Or, la courbe de la répartition est assez différente de celle des probabilités, bien connue des statisticiens sous le nom de courbe des erreurs ou de Gauss. Pareto en conclut que la forme de la pyramide ou de la toupie renversée est déterminée par des forces fondamentalement indépendantes, régies presque certainement par une loi universelle. Les pauvres occupent la partie inférieure, arrondie, de la pyramide ou de la toupie renversée, tandis que les riches occupent la partie supérieure, pointue. L'augmentation des bas revenus et donc une diminution des inégalités ne peuvent se vérifier, soit 'isolément soit cumulativement, que si le total des revenus ne crôit pas plus vite que l'augmentation de la population. Ni l'accroissement général de la richesse ni l'accroissement du nombre des personnes qui ne possèdent rien n'impliquent nécessairement ni une augmentation ni une diminution de la richesse globale de la société. Ceci montre que l'inégalité des fortunes et la diminution du paupérisme sont deux choses bien distinctes. (Pareto, 1965: 43-48).

La structure de l'objet scientifique construit et appelé courbe des revenus, n'est donc rien d'autre qu'une description/représentation fondée sur une théorie, de laquelle on déduit également qu'une distribution différente de la richesse peut élargir la base de la pyramide ou de la toupie renversée, en restreindre le sommet, mais pas du tout éliminer les rapports d'inégalité, qui demeurent fondamentalement stables dans le temps. L'inégalité ne dépendrait pas de l'organisation économique de la société, mais plutôt de facteurs naturels, de la nature humaine, qui fixeraient une fois pour toujours la constance de l'ordre social.

Le modèle construit par Pareto est une structure. Au delà de la représentation manifeste du directement observable (les riches et les pauvres dans une société), il révèle un ordre latent déductible indirectement (Barbut, 2007: 156-166).

\section{FORME ET FOND}

Les phénomènes sociaux se présentent, pour Pareto, selon des formes changeantes, manifestées par les idéologies, les coutumes, les représentations collectives, la pensée consciente, le vocabulaire, la conceptualisations des sentiments, en bref par tous les systèmes symboliques. Par contre, le fond est révélé exclusivement de manière déductive par l'analyse théorique, qui, à travers l'étude des relations, montre la signification de cet ordre rationnel latent. Par exemple, les 
doctrines sur la répartition de la richesse sont la forme changeante du phénomène. Le fond est constitué par la structure de la répartition: celle-ci indique que les sociétés sont hétérogènes et que l'inégalité est le trait constant de tout ordre social quel qu'il soit. Cette distinction recouvre des caractéristiques différentes selon qu'il s'agit de la dualité résidu-dérivation, histoire longue durée-événement singulier.

La forme et le fond constituent, d'un autre point de vue, l'aspect subjectif et l'aspect objectif à travers lesquels les phénomènes se présentent: l'aspect subjectif est la forme par laquelle l'esprit humain se représente les phénomènes, représentation souvent déformée, tandis que l'aspect objectif est le substrat constant, non changeant. Le fond est le constant, le général, l'essentiel. La forme est le variable, le singulier, l'occasionnel.

Les phénomènes sont une trame d'actions humaines et de relations entre ces actions. Les phénomènes sociaux sont des conduites humaines. C'est de ces unités fondamentales très complexes et compliquées qu'il faut partir pour expliquer la production de la société, conçue comme un système d'actions interdépendantes, dont l'analyse révèle les uniformités. Une première constatation montre que les actions sociales peuvent être distinguées en deux grandes catégories: les actions logiques qui «sont au moins dans leur partie principale, le résultat d'un raisonnement» et les actions non-logiques qui «proviennent principalement d'un certain état psychique: sentiments, subconscience, etc.» (Pareto, 1968: § 161).

Les valeurs, les intérêts, les sentiments, les idéologies, les croyances, les résidus, jouent un rôle capital dans les conduites des hommes et dans leurs actions. «Les phénomènes sociaux sont principalement déterminés par les sentiments et les intérêts, et seulement d'une manière très secondaire par des raisonnements logiques et expérimentaux. D'autres raisonnements interviennent, au contraire, très largement. Ils comprennent les sophismes logiques, mais ceux-ci n'en sont que la moindre partie, la plus grande est constituée par des développements qui s'accordent simplement avec certains sentiments, certains intérêts.» (Pareto, 1984: 309). En d'autres termes, les choses changent dans leurs formes sans modification du fond. Ce fond serait donné par la relative identité constante de la nature humaine dans le temps et dans l'espace (Pareto, 1978: I, 130-134; Pareto, 1965: 43-48).

\section{ACTIONS LOGIQUES ET ACTIONS NON-LOGIQUES}

Les actions logiques sont celles qui utilisent des moyens appropriés au but et unissent logiquement les moyens au but; les actions non-logiques sont celles où la connexion logique entre les moyens et les buts est inexistante. Pour qu'une action soit effectivement logique, il ne suffit pas qu'il y ait une connexion, pour l'acteur, entre l'action et le but; il faut que cette connexion existe aussi pour «ceux qui ont des connaissances plus étendues » (Pareto, 1968: § 150). Dans ce cas, l'action est logique soit objectivement (le tiers ayant des connaissances étendues) soit subjectivement (la personne agissant). Les premières se servent du matériel expérimental et de faits objectifs établis et unis entre eux par des raisonnements rigoureux; elles fournissent des objets scientifiques à des disciplines sectorielles 
comme l'économie, l'histoire, le droit, etc., où les critères de la vérité empirique et de la validité logique prédominent. Les secondes, de loin les plus nombreuses, ont une grande importance dans la vie sociale et circulent presque toujours plus ou moins colorées de logique. Ces actions non-logiques sont généralement un «tas d'absurdités » (Pareto, 1968: § 445), elles reflètent l'arbitraire et le changeant des manières de penser et d'agir des hommes, le poids coercitif du milieu, le rôle des interactions, les effets de l'agrégation des comportements individuels, aussi bien que la présence en chacun de nous de préjugés, de croyances, de valeurs, d'éthos. La socialisation intègre tout cela en des systèmes symboliques très stables et souvent institutionnalisés: dans les deux cas, l'action est cristallisée en des structures significatives intersubjectivement objectives. En effet, l'étude analytique des processus de pensée, des procédures d'argumentation, de la relation entre les valeurs visées intentionnellement et les sentiments inscrit dans la nature humaine, des croyances collectives et de l'unité du moi, tout cela permet de distinguer les rapports de signe à chose signifiée des rapports de causalités, les paralogismes de composition des paralogisme de division, et donne à la recherche un point fort d'accostage, ni exclusivement extérieur ni essentiellement intérieur. A ce propos Pareto écrit: «Les actions non-logiques sont généralement considérées au point de vue logique par ceux qui les accomplissent ou par ceux qui en traitent, qui en font la théorie. De là, la nécessité d'une opération de prime importance pour notre étude, laquelle tend à lever ces voiles et à retrouver les choses qu'ils dissimulaient. C'est aussi contraire à beaucoup de théories qui s'arrêtent aux voiles, non tenus pour tels, mais pris pour la partie fondamentale des actions. "Nous devrons examiner ces théories; parce que si nous les trouvions vraies - c'est-à-dire d'accord avec l'expérience -, nous devrions prendre une tout autre voie que celle qu'ils conviendrait de suivre, si nous reconnaissions que la partie fondamentale est au contraire la chose voilée». Et il ajoute aussitôt après: "La vérité expérimentale d'une théorie et son utilité sociale sont des choses différentes. Une théorie expérimentale vraie peut être utile - ou nuisible - à la société, comme aussi une théorie expérimentalement fausse.» (Pareto, 1968: § 249). Dès lors seule la «logique des sentiments» rend intelligible les coutumes, les mœurs, les conduites des acteurs sociaux, les agissements des organisations sociales et des institutions. Les sentiments façonnent les actions et les comportements, ils sont plus importants et déterminants que les raisonnements et les arguments avec lesquels ils ont été habillés. Puisqu'en logique le rapport moyen-fin est essentiel, Pareto n'a aucune difficulté à admettre une rémanence non-logique même dans les actions logiques. La logique qualifie le rapport moyen-fin mais elle est impuissante à déterminer et fonder logiquement les fins.

En analysant et en comparant les raisonnements et les développements logiques ajoutés aux actions non-logiques, Pareto retrouve la face latente et la face manifeste. La première est stable, la seconde est variable. La première est prédonnée, elle fuit toute explication empirique et peut être seulement conceptualisée par le biais de déductions du système-structure symbolique, tandis que la seconde est fonction des contingences et elle est toujours observable empiriquement. La face constante et latente est appelée résidus; la face manifeste et variable, par contre, est appelée dérivations.

Cette approche trouve quelques échos dans ce que la sociologie contemporaine dénomme habitus, c'est-à-dire «systèmes de dispositions durables, structures 
structurées, prédisposées à fonctionner comme structures structurantes, c'est-àdire en tant que principe de génération et de structuration de pratiques et de représentations qui peuvent être objectivement 'réglées' et 'régulières' sans être en rien le produit de l'obéissance à des règles, objectivement adaptées à leur but sans supposer la visée consciente des fins et la maîtrise expresse des opérations nécessaires pour les atteindre et, étant tout cela, collectivement orchestrées sans être le produit de l'action organisatrice d'un chef d'orchestre» (Bourdieu, 1972: 175).

\section{RÉSIDUS ET DÉRIVATIONS}

Les résidus ne sont ni les instincts, ni les sentiments, ni les inclinaisons biologiques. Ils n'existent pas; il s'agit d'une entité hypothétique en vue d'expliquer les actions et les expressions linguistiques relatives. Ils sont des structures avec des significations symboliques. Ces structures ne sont pas observables directement; ce sont des objets scientifiques pareils aux radicaux de la langue. Déterminer ce qui correspond effectivement à cet état psychique fondamentale, c'est une tâche étrangère à la sociologie: «Dans notre étude, nous partons de cet état de fait, sans vouloir remonter plus haut» (Pareto, 1968: § 161). Cet état fondamental est constitué par les résidus interdépendants les uns des autres. Ils expriment les aspects normatifs et conditionnels du système d'action. Irréductibles aux impulsions et aux instincts de la psychologie antirationaliste, les résidus sont des prémisses, des principes universels (métaphysiques, moraux, juridiques, politiques, etc.) au sens logique; ils possèdent des contenus cognitifs. Le concept de résidu dépasse l'approche subjective car il réussit à englober l'intention et la symbolisation de l'intention. De cette façon, «les sentiments, la subconscience, etc.», s'étant transfigurés en des rapports symboliques, deviennent des objets intelligibles et compréhensibles, donc accessibles. Les dérivations, par contre, sont le produit de l'expérience concrète; elles fournissent, avec la médiation de techniques argumentatives, les biais pour le rétablissement, l'intégrité d'un tout, des ensembles. De même elles livrent les argumentations susceptibles d'expliquer et faire valoir l'agir. Elles précèdent les sentiments et contribuent même à les renforcer: "Les résidus sont des manifestations de sentiments. Les dérivations comprennent des raisonnements logiques, des sophismes, des manifestations de sentiments employées pour dériver; elles sont une manifestation du besoin de raisonner qu'éprouve l'homme. Si ce besoin n'était satisfait que par les raisonnements logico-expérimentaux, il n'y aurait pas de dérivations et à leur place, on aurait des théories logico-expérimentales. Mais le besoin de raisonnement de l'homme trouve à se satisfaire de beaucoup d'autres manières: par des raisonnements pseudo-expérimentaux par des paroles qui excitent les sentiments, par des discours vains et inconsistants; ainsi naissent les dérivations. Elles font défaut aux deux extrêmes: d'une part pour les actions instinctives, d'autre part, pour les sciences rigoureusement logico-expérimentales. On les rencontre dans les cas intermédiaires » (Pareto, 1968: $§ 1401)$.

Une tendance exagérée à simplifier, un goût prononcé pour la polémique qu'un tempérament passionnel peut expliquer mais point légitimer, poussent Pareto à élaborer une typologie et une classification des résidus et à la présenter avec une audace qui déconcerte, notamment il affirme que les manifestations des 
sentiments doivent être considérées en tant que forces déterminant l'équilibre social. Cet hypersensible, cet homme aux passions sans affection, ce solitaire sans tendresse, fait et défait, sans interruption, des typologies et des classifications, sans se préoccuper ni de l'ordre ni de la cohérence de ses constructions théoriques ni même de différencier les rapports généalogiques des rapports logiques. Le statut des justifications des mobiles des actions est conçu de façon analogue à celui des idées concernant les modalités et les moyens pour les accomplir.

Les résidus constituent six classes, décomposées en six genres: (a) l'instinct des combinaisons; (b) la persistance des agrégats; (c) le besoin de manifester ses sentiments pour des actes extérieurs; (d) les résidus en rapport avec la sociabilité; (e) l'intégrité de l'individu et de ses dépendances; (f) le résidu sexuel. Ces genres varient au cours des étapes du développement social, cependant les variations se compensent toujours, de sorte que les classes restent toujours constantes. Par ailleurs, ces classes, tout en étant hétérogènes, contiennent les éléments indispensables pour assurer la constance de l'ensemble et cela malgré les variations de détail. L'affinité génétique entre les tendances affectives ou intellectuelles, recensées en une même catégorie, n'est pas démontrée ni même argumentée. Par ailleurs, il est impossible de déterminer l'abondance relative des résidus de l'une ou de l'autre classe dans un phénomène social donné. La constance des résidus est présupposée pour obtenir une espèce d'infrastructure susceptible de fonder la causalité de l'action. Mais celle-ci n'est pas réalisable régulièrement à partir du moment où certains résidus sont des normes ou des valeurs cristallisées, des produits historiques d'interactions. Il ne fait pas de doute, donc, que le problème des rapports entre la causalité des conduites et les implications des représentations reste irrésolu car il n'est pas dit quand certains résidus sont constants et quand c'est à l'interprète à les concevoir ainsi.

Les discours, les théories pseudo-scientifiques, les idéologies en général (et Pareto considère tel tout le normatif) ne sont qu'un simple reflet des intérêts réels, reflet qui est dénommé, comme on l'a déjà dit, dérivations. «Les hommes se laissent persuader surtout par les sentiments (résidus); par conséquent, nous pouvons prévoir, ce qui d'ailleurs est confirmé par l'expérience, que les dérivations tireront leur force, non pas de considérations logico-expérimentales, ou du moins pas exclusivement de ces considérations, mais bien des sentiments. Dans les dérivées, le noyau principal est constitué par un résidu ou par un certain nombre de résidus. Autour de ce noyau viennent se grouper d'autres résidus secondaires. Cet agrégat est créé par une force puissante, et quand il a été créé, il est maintenu uni par cette force, qui est le besoin de développements logiques ou pseudo-logiques qu'éprouve l'homme, besoin qui se manifeste par les résidus du genre (I-e). C'est ensuite de ces résidus avec l'aide d'autres résidus du genre. C'est ensuite de ces résidus avec l'aide d'autres encore, que les dérivations tirent en général leur origine» (Pareto, 1968: $§ 1397)$. Comme les résidus, les dérivations aussi sont rangées en classes et en genres. Les classes sont au nombre de quatre: (a) affirmation; (b) autorité; (c) accord avec des sentiments ou avec des principes; (d) preuves verbales.

Les dérivations sont fondées sur le langage. Celui-ci donne substance et apparence à toute chose, lui confère ordre et grandeur, lui fixe un sens. En d'autres mots, le langage est tout: par le biais du langage, les résidus révèlent la rationalité sociale, la logique des sentiments, la structure des actions et les lois relatives 
d'organisation et d'équilibre. Les dérivations dissimulent les résidus, structurent leurs expressions ainsi que leurs représentations. La théorie des dérivations révèle la logique des sentiments et ouvre le chemin à la «nouvelle rhétorique », aux théories de l'argumentation, aux théories des logiques non démonstratives ainsi qu'à l'étude des idéologies.

Pareto réserve une place de choix à l'analyse des idéologies, aux raisons pour lesquelles les agents sociaux attribuent toujours des motifs rationnels à leurs agissements, pourquoi ils fournissent des explications ou des justifications posthumes à ce ils font ou croient faire. Les idéologies sont des expressions de ce que les agents sociaux désirent ou craignent, de ce qu'ils apprécient ou désapprouvent, de ce qu'ils pensent faire ou ne pas faire. Tantôt elles dissimulent les résidus tantôt elles les voilent. Assemblage composite de facteurs cognitifs et affectifs, de causalité et de ressemblance, les idéologies font coexister l'irréductibilité des jugements de valeur et des jugements de fait avec l'efficacité et l'utilité sociales.

L'articulation étroite de la logique, de la psychologie et de la rhétorique donne lieu aux modèles du masquage/déguisement, de l'expression figurée, de l'engendrement et de la couverture, dont le maniement, assurément, est périlleux. Pareto ne cherche pas à mettre en évidence des catégories fondamentales, il veut seulement indiquer comment sont engendrées, grâce à un noyau psychologique commun, les croyances, les intentions des acteurs et les raisonnements au moyen desquels on les explicite. Pour cette raison il rappelle que «Dans les sciences sociales, il faut surtout se tenir sur ses gardes contre l'intromission des sentiments de l'auteur, lequel incline à rechercher, non simplement ce qui existe, mais ce qui devrait exister pour concorder avec ses sentiments religieux, moraux, patriotiques, humanitaires ou autres. La recherche des uniformités expérimentale est en elle-même un but. Quand on a trouvé ces uniformités, elles peuvent servir à d'autres buts; mais confondre ces deux recherches porte un grave préjudice à toutes les deux. [...]. Si donc nous voulons ramener les sciences sociales au type des sciences naturelles, il faut que nous procédions [...], en réduisant les phénomènes concrets très compliqués à des phénomènes théoriques beaucoup plus simples, en nous laissant guider dans cette opération exclusivement par l'intention de découvrir des uniformités expérimentales, et en jugeant leur efficacité uniquement par les vérifications expérimentales que nous pouvons faire.» (Pareto, 1968: $\S 2411)$.

\section{PROPRIÉTÉS DES RÉSIDUS ET DES DÉRIVATIONS}

Les résidus et les dérivations font connaître les manifestations de certaines forces qui œuvrent sur la forme que prend la société. Comment ces agrégats œuvrent-ils? Si leur rapport avec la réalité est arbitraire, où la fonction de ces systèmes symboliques s'enracine-t-elle? En d'autres mots, «En quel rapport cette action est-elle avec l'utilité sociale ?» (Pareto, 1968: § 1687).

Pareto examine d'abord, pour la partie statique, la répartition des résidus dans une société donnée et dans les diverses strates de cette même société. Ensuite, pour la partie dynamique, il étudie comment les résidus varient dans le temps, soit qu'ils changent chez les individus d'une même strate sociale, soit que le change- 
ment s'opère à cause du mélange des strates sociales entre elles, sans omettre l'étude suivant laquelle chacun de ces phénomènes se déroule et comment il agit. La propagation des résidus et des dérivations se fait par imitation ou à cause d'autres circonstances qui œuvrent sur la collectivité.

Pareto ne donne pas une théorie de la diffusion et de la communication sociale, cependant l'étude qu'il fait des processus de propagation met en évidence l'existence d'un troisième élément: les intérêts. «Les individus et les collectivités sont poussés par l'instinct et par la raison à s'approprier les biens matériels utiles, ou seulement agréables à la vie, ainsi qu'à rechercher de la considération et des honneurs. On peut donner le nom d'intérêts à l'ensemble de ces tendances. Cet ensemble joue un très grand rôle dans la détermination de l'équilibre social» (Pareto, 1968: § 2009).

Cette notion d'intérêt reste ambiguë. En effet, tantôt il s'agit d'une catégorie d'action déterminée par les résidus «intégrité de l'individu et ses dépendances» tantôt elle renvoie à des actions propres à l'«homo oeconomicus ».

Conditions d'intelligibilité de l'action, dépourvus d'existence objective, liés entre eux par la mutuelle dépendance ou par la causalité multiple, les résidus, les dérivations et les intérêts, facteurs nécessaires de l'équilibre général, ne peuvent toutefois jamais être saisis dans leur intégralité.

La méthode est-elle donc défaillante ? La réponse de Pareto est: «non, parce que nous en tirons notamment deux grands avantages: $1^{\circ} \mathrm{Il}$ donne à notre esprit une image des phénomènes, image que nous ne pourrions obtenir d'aucune autre façon $[\ldots] .2^{\circ}$ Il nous indique la voie que nous devons suivre pour éviter les erreurs du procédé (1), et pour nous rapprocher de la réalité [...]. $3^{\circ}$ Enfin, la notion, même imparfaite, de la mutuelle dépendance, nous engage » et nous aide à éviter la méthode basée sur une seule structure causale. Et grâce à la méthode imparfaite de la causalité multiple, on sait justement «que les résidus étaient beaucoup plus constants que les dérivations; c'est pourquoi nous avons pu considérer qu'ils étaient en partie la cause des dérivations, mais sans oublier l'action secondaire des dérivations, qui peuvent être parfois la cause des résidus [...]» (Pareto, 1968: $\S$ 1732). Avec d'autres termes, il s'agit d'un système de relations, presque une matrice de perceptions et d'appréciations grâce à laquelle on fait la médiation entre les structures objectives intériorisées et les conduites individuelles. Cette matrice varie de société à société, d'une classe sociale à l'autre, d'un groupe culturel à un autre. Les pourquoi de ces variations et leur composition dans les différents cas d'espèce ne sont pas explicités nettement.

\section{L'ÉQUILIBRE SOCIAL}

La société est composée de différents éléments interdépendants: le sol, le climat, la faune, la flore, les actions des autres sociétés sur elle, l'histoire, la race, les résidus, les dérivations, les dérivés, les intérêts. Pareto précise opportunément: «En tout cas, que le nombre des éléments que nous considérons soit petit ou grand, nous supposons qu'ils constituent un système [...]. Ce système change de forme et de caractère avec le temps; et quand nous nommons le système social, nous entendons ce système considéré aussi bien en un moment déterminé, que dans les transformations successives qu'il subit en un espace de temps déterminé.» (Pareto, 
1968: § 2066). Société, donc, en tant qu'ensemble symbolique complet, à l'intérieur duquel chaque unité contribue à former le tout et qui peut être analysé si nous fixons l'état où nous voulons considérer le système social. Or «Il change à chaque instant». (Pareto, 1968: § 2069).

Le modèle proposé pour analyser l'équilibre social est raccordé étroitement aux résidus et aux dérivations, aux représentations conçues comme des principes dont on peut déduire logiquement des conclusions.

Pour étudier les phénomènes dynamiques, il faut maintenir inchangé l'état $X 1$ au temps $t 1$ et l'état $X 2$ au temps $t 2$ et ainsi de suite l'état $X n$ au temps $t n$. "L'état $X$ que nous considérons est semblable à celui d'un fleuve; les états $X l, X 2, \ldots$, sont semblables à ceux de ce fleuve, chaque jour, par exemple. Le fleuve n'est pas immobile; il coule, et toute modification, si petite soit-elle, qu'on apporte à sa forme et à son cours, est la cause d'une réaction qui tend à rétablir l'état primitif.» (Pareto, 1968: § 2071). L'état $X$ est tel «que si l'on y introduisait artificiellement quelque modification différente de celle qu'il subit en réalité, aussitôt se produirait une réaction qui tendrait à le ramener à l'état réel. Ainsi, l'état $X$ est rigoureusement défini.» (Pareto, 1968: 2068). C'est l'état d'un phénomène déterminé par les conditions qui l'ont engendré, c'est en bref un processus de changement non casuel, non simplement stochastique. Le point où la régulation atteint un état d'équilibre est défini par le moyen d'un ensemble d'égalités simples qui coïncident seulement d'une manière momentanée avec un système d'opérations réversibles. Ici aussi le système se base sur la substitution des égalités et sur l'entière conservation du tout. Du moment que les formes supérieures d'implication, c'està-dire les normes morales et juridiques, n'ont aucun poids sur l'équilibre social, si ce n'est qu'à titre de véhicules de sentiments instinctifs, Pareto doit postuler, en définitive, la simultanéité des actions et des effets, l'exclusion de l'effet des prévisions individuelles et collectives, la réciprocité et la réversibilité des liens entre tous les facteurs de l'équilibre, et même la continuité des grandeurs et l'unicité des solutions. L'accent doit, par conséquent, être mis sur les relations d'interdépendance. Evidemment, on ne trouve dans l'œuvre de Pareto aucune préoccupation pour les fins, soient-ils ultimes, qui ne sont pas déterminables. Malgré cela, la question du changement qui s'introduit dans l'évolution reste légitime. Si l'on écarte, comme le fait Pareto, les finalités extérieures, on ne peut exclure la réduction de la finalité à la conscience de l'équilibration. Cette dernière ne signifie pas marche obligatoire vers l'homogène, mais plutôt coordination entre des tendances différenciées, coordination qui produira un système de transformation. On sait que ces systèmes de transformation peuvent être parfaitement mobiles, parfaitement différenciés, et en même temps permanents, c'est-à-dire actifs dans le temps.

Hétérogène à cause des éléments qui déterminent l'état d'équilibre, en mouvement ondulatoire sous l'aspect dynamique, le système social est ainsi caractérisé par une dimension synchronique et une dimension diachronique. Forme et fond, aspect latent et aspect manifeste, aspect subjectif et aspect objectif, vérité et utilité, intention et signification, statique et dynamique structurent un modèle d'analyse où le causalisme mécaniste coexiste avec le naturalisme, l'intentionnalité et l'actionnisme. Ainsi Pareto explique les comportements sociaux grâce tantôt aux causes psychologiques et biologiques tantôt aux contextes des actions, aux intentions, aux motivations, aux raisons conscientes des individus. En allant au-delà des acteurs sociaux, l'explication est ancrée dans le substrat infra-individuel. 


\section{L'HÉTÉROGÉNÉITÉ SOCIALE}

La société est la résultante d'éléments divers et disparates, de forces concurrentes ou antagonistes, d'institutions diverses, de différentes façons d'intérioriser les contraintes sociales; elle est le lieu des conflits et des rivalités. L'équilibre y est continuellement précaire. Cela est dû au fait que la société n'est pas homogène; elle est composée de groupes différents et rivaux, à cause de l'âge, du sexe, de la force physique, de la santé, etc. Les individus et les groupes, pour obtenir le maintien ou la réalisation d'un but ou pour satisfaire un besoin réel ou hypothétique, se servent opportunément des circonstances externes, des sentiments, des intérêts et même des dérivations comme moyen de propagande.«Les raisonnements logicoexpérimentaux ont une grande valeur, lorsque le but est donné et que l'on cherche les moyens propres à l'atteindre. Par conséquent, ils sont employés avec succès dans les arts et métiers, en agriculture, dans l'industrie, dans le commerce. Ainsi, à côté de nombreuses sciences techniques, on a pu constituer une science générale des intérêts, l'économie, qui suppose ces raisonnements employés exclusivement dans certaines branches de l'activité humaine. Ces raisonnements trouvent aussi leur application à la guerre, et ont donné naissance à la stratégie et à d'autres sciences semblables. Ils pourraient aussi s'appliquer à la science du gouvernement; mais, jusqu'à présent, ils ont été employés comme arts individuels de gouverner, plutôt que pour constituer une science abstraite; cela parce que le but n'est pas déterminé, ou que, s'il est déterminé, on ne veut pas le dévoiler. En général, pour ces motifs et pour d'autres, les raisonnements logico-expérimentaux ont joué un rôle effacé dans l'organisation de la société. Il n'y a pas encore de théories scientifiques en cette matière, et pour tout ce qui s'y rattache, les hommes sont mus beaucoup plus par les sentiments que par les raisonnements. Un certain nombre de personnes savent tirer profit de cette circonstance et s'en servir pour satisfaire leurs intérêts; ce faisant, de temps à autre, elles utilisent opportunément des raisonnements en partie empiriques et en partie logico-expérimentaux.» (Pareto, 1968: § 2146).

Ce qu'un individu ou un groupe estime profitable ou concevable pour se procurer ceci ou cela, peut-il être dit utile? Existe-t-il une utilité pour l'individu et une autre pour la société? Si les utilités individuelles sont disparates, l'utilité collective peut-elle être homogène ? Pareto est catégorique: «Pour avoir une idée plus précise, il est nécessaire d'énoncer les normes, en partie arbitraires, que l'on entend suivre pour déterminer les entités que l'on veut définir. L'économie pure a pu le faire: elle a choisi une norme unique, soit la satisfaction de l'individu, et a établi qu'il est l'unique juge de cette satisfaction. C'est ainsi qu'on a défini l'utilité économique ou ophélimité. Mais si nous nous posons le problème, très simple aussi, de rechercher ce qui est le plus profitable à l'individu, abstraction faite de son jugement, aussitôt apparaît la nécessité d'une norme, qui est arbitraire.» (Pareto, 1968: § 2110). Si les utilités étaient homogènes, et si on pouvait donc les comparer et les sommer, on n'aurait à faire à aucune difficulté. Malheureusement «Les utilités des divers individus sont des quantités hétérogènes, et parler d'une somme de ces quantités n'a aucun sens; il n'y en a pas: on ne peut l'envisager. Si l'on veut avoir une somme qui soit en rapport avec les utilités des divers individus, il est nécessaire de trouver tout d'abord un moyen de faire dépendre ces utilités de quantités homogènes, que l'on pourra ensuite additionner.» (Pareto, 1968: 
$\S$ 2127). L'opposition des utilités, l'opposition des intérêts, la division de la société, des valeurs différentes, entraînent des divergences de buts, des effets non voulus et pourtant réalisés, des effets voulus et non réalisés. Voilà le fondement de l'hétérogénéité, voilà pourquoi les effets significatifs, intentionnels et non évidents des actions ne produisent pas nécessairement une rationalité sociétale. «De là nous devons conclure, non pas qu'il est impossible de résoudre des problèmes qui considèrent en même temps différentes utilités hétérogènes, mais bien que, pour traiter de ces utilités hétérogène, il faut admettre quelque hypothèse qui les rende comparables. Lorsque cette hypothèse fait défaut, ce qui arrive très souvent, traiter de ces problèmes est absolument vain; c'est simplement une dérivation dont on recouvre certains sentiments, sur lesquels seuls, par conséquent, nous devrons fixer notre attention, sans trop nous soucier de leur enveloppe.» (Pareto, 1968: § 2137).

Tout rend donc la société hétérogène. Il est impossible de parler d'une rationalité intrinsèque de la société. Pourtant, il est possible de donner une explication rationnelle de la non rationalité sociale. Dés lors peut-on dire que l'hétérogénéité, la division de la société, les divergences de valeurs seront un jour surmontés? Pareto répond sans équivoque: non. «N'en déplaise aux humanitaires et aux positivistes, une société déterminée exclusivement par la raison n'existe pas et ne peut exister; et cela, non parce que les 'préjugés' des hommes les empêchent de suivre les enseignements de la 'raison', mais parce que les données du problèmes que l'on veut résoudre par le raisonnement logico-expérimental font défaut.» (Pareto, 1968: $\S \S 1878,1880$ à 1882). Ici apparaît de nouveau «l'indétermination de la notion d'utilité [...]. Les notions que les différents individus ont au sujet de ce qui est bien pour eux-mêmes ou pour autrui sont essentiellement hétérogènes, et il n'y a pas moyen de les réduire à l'unité.» (Pareto, 1968: § 2143). Il ne faut pas oublier que l'homme «n'est pas un être de pure raison, c'est aussi un être de sentiment et de foi, et le plus raisonnable ne peut se dispenser de prendre parti, peut-être même sans en avoir nettement conscience, au sujet de quelques-uns, au moins, des problèmes dont la solution dépasse les bornes de la science.» (Pareto, 1978: I, 2-3).

Les actions des hommes sont animées par des mobiles affectifs, les motifs rationnels qu'on en donne sont des rationalisations à posteriori, sont des pseudoraisons, des raisonnements qui dissimulent d'autres représentations ou affects, des raisonnements biaisés ayant l'apparence du vrai. Pareto ne dénie pas l'existence de motifs rationnels chez les acteurs, il ne s'intéresse pas à l'universalité des catégories et aux a priori de l'esprit humain. Sa sollicitude analytique s'adresse surtout aux procédures des raisonnements, aux constantes des argumentations, à la psychologie des catégorisations. Il quête les universaux argumentatifs communs aux agents interagissant les unes avec les autres, les mécanismes d'autopersuasion, le rôle des paralogismes dans les fausses croyances sur le social, les effets agrégés paradoxaux de l'action individuelle et collective.

\section{MOUVEMENT ONDULATOIRE ET ÉVÉNEMENTS HISTORIQUES}

Dans la mesure où les résidus se transforment lentement, les sociétés aussi changent. Certes, il y a une part toujours plus grande de la raison dans l'activité 
humaine, mais cette proposition reste indéfinie et donne facilement lieu à plusieurs erreurs. Ce progrès se fait en suivant un mouvement ondulatoire ou rythmique. Les oscillations ou rythmes ont une ampleur, une durée et une intensité diverses. «En réalité, les oscillations des diverses parties du phénomène social sont en rapport de mutuelle dépendance, à l'égal de ces parties mêmes; elles sont simplement des manifestations des changements de ces parties. Si l'on tient à se servir du terme fallacieux de cause, on peut dire que la période descendante est la cause de la période ascendante qui la suit, et vice-versa. Mais il faut entendre cela uniquement en ce sens que la période ascendante est indissolublement unie à la période descendante qui la précède, et vice-versa. En conséquence, les différentes périodes sont seulement des manifestations d'un seul et unique état de choses, elles se succèdent les unes aux autres. Cette succession est une uniformité expérimentale. Il existe divers genres de ces oscillations, selon le temps où elles se produisent. Ce temps peut être très court, court, long, très long. Les oscillations très courtes sont habituellement accidentelles, en ce sens qu'elles manifestent des forces peu durables; celles qui se produisent en un temps assez long manifestent habituellement des forces assez durables (Pareto, 1968: § 2331). «Etant donné que nous connaissons mal des temps très reculés, et vu l'impossibilité où nous sommes de prévoir l'avenir, les oscillations très longues peuvent perdre le caractère d'oscillation, et apparaître comme manifestant un cours qui se dirige toujours dans la même direction » (Pareto, 1968: $\$ § 2338$ et 2392).). Quand un phénomène atteint sa plus forte intensité, c'est l'oscillation en sens contraire qui est en général proche. D'où l'impossibilité d'expliquer les phénomènes sociaux en employant une causalité simplement linéaire ou un déterminisme plus ou moins rigide. Qu'on prenne le cas des révolutions politiques, sociales, religieuses. Elles sont justes, bienfaisantes, nécessaires pour les uns; injustes, mauvaises, inutiles pour les autres. "Au point de vue scientifique cela n'a pas de sens. Une proposition scientifique est vraie ou est fausse, elle ne peut en outre satisfaire à une autre condition [...]. La science ne s'occupe que de constater les rapports des choses, des phénomènes, et de découvrir les uniformités que présentent ces rapports. L'étude de ce qu'on appelle des causes, si par là on entend des faits en certains rapports avec d'autres, appartient à la science et rentre dans la catégorie précédente des uniformités. Mais ce que l'on a appelé les causes premières, et en général toutes les entités qui dépassent les bornes de l'expérience, se trouvent par là même en dehors du domaine de l'expérience.» (Pareto, 1978: I, 2).

La plus importante de ces uniformités du domaine de l'expérience est, assurément, celle concernant la circulation des élites.

\section{ÉLITES ET CIRCULATION DES ÉLITES}

Les sociétés ne sont pas homogènes. Elles sont divisées en groupes et classes assez hétérogènes, mais la séparation n'est pas absolue. Il existe à l'intérieur des groupes et des classes et entre les groupes et les classes une circulation intense, verticale et horizontale. Les groupes et les classes sont en conflit et il ne fait pas de doute que la lutte des classes est un élément déterminant dans la vie des sociétés. Il existe également une lutte à l'intérieur des groupes et des classes pour obtenir l'hégémonie sur ces groupes et sur ces classes. La partie du groupe ou de 
la classe qui essaie de s'assurer l'hégémonie sur son propre groupe ou sur sa propre classe, ou aussi sur tous les groupes et sur toutes les classes de la société, est appelée élite. Il n'y a, donc, pas d'incompatibilité entre la théorie de la lutte de classe et la théorie des élites. Cette dernière prétend être une généralisation de la première.

Pour Pareto, à l'intérieur d'une classe comme à l'intérieur de la société, il y a des personnes qui manifestent de grandes capacités dans les branches respectives de l'activité sociale. L'ensemble de ces gens-ci est regroupé en une classe à laquelle on donne le nom d'élite. Mis à part «ceux qui, directement ou indirectement, jouent un rôle notable dans le gouvernement [et qui] constitueront l'élite gouvernementale», le «reste formera l'élite non gouvernementale» (Pareto, 1968: $§ 2032$ ). «Nous avons donc deux couches dans la population: $1^{\circ}$ la couche inférieure, la classe étrangère à l'élite; nous ne recherchons pas, pour le moment, l'influence qu'elle peut exercer dans le gouvernement; $2^{\circ}$ la couche supérieure, l'élite, qui se divise en deux: (a) l'élite gouvernementale; $(b)$ l'élite non gouvernementale» (Pareto, 1968: § 2034). Ailleurs Pareto clarifie mieux ce schéma analytique de la classe supérieure et de la classe inférieure ainsi: «Le moins que nous puissions faire est de diviser la société en deux couches: une couche supérieure, dont font habituellement partie les gouvernants, et une couche inférieure, dont font partie les gouvernés. Ce fait est si manifeste qu'il s'est en tout temps imposé à l'observateur le moins expert; il en est de même du fait de la circulation des individus entre ces deux couches.» (Pareto, 1968: § 2047).

Cette stratification de la société, corroborée aussi par la théorie de la distribution de la richesse, est fondée sur la nature des hommes, elle n'est pas le produit de forces économiques ou de capacités organisationnelles spéciales. En bref, l'inégalité (car au fond, on ne parle que de cela) entre les hommes est déterminée par la possession de certaines qualités psychologiques. Ces qualités personnelles font que certains hommes cherchent et obtiennent l'hégémonie et que d'autres doivent nécessairement la subir. Que le rôle de commandement revienne toujours à une minorité est pour Pareto une banalité que même les institutions représentatives n'arrivent pas à détruire. Le seul vrai problème est celui qui part de l'uniformité historique du processus de constitution, de formation, de transformation, de mort et de substitution de cette minorité. L'étude de cet acteur historique, de sa circulation, de sa rotation et de ses successions, constitue le point focal dans l'étude du système social.

Qu'est-ce que l'élite? Quels caractères et quelles qualités la distinguent-ils? Comment se forme-t-elle? Comment dégénère-t-elle? Quelles fonctions remplitelle?

Pour Pareto «ceux qui ont les indices les plus élevés dans la branche où ils déploient leur activité» (Pareto, 1968: § 2031) composent l'élite. Ces indices montrent l'existence de certaines capacités dans l'exercice et dans la pratique de n'importe quelle activité humaine. Les capacités sont pour Pareto la disposition naturelle de l'individu à exceller dans une activité déterminée. Cette disposition naturelle, qu'aujourd'hui les sciences sociales appellent attitudes, est donnée essentiellement par l'hérédité. Or, en faisant abstraction du fait que rien n'a prouvé jusqu'à présent l'existence de dons naturels, on ne peut nier que la supériorité fournie par la possession des «capacités» est telle en raison du fait que certaines normes sociales la valorisent. Ce n'est pas l'existence de certaines 
« capacités » qui détermine la supériorité, mais bien le fait qu'un groupe social, ou une société, décide de valoriser telle ou telle capacité. Pourquoi, d'autre part, le groupe choisit certaines valeurs à la place d'autres, c'est un fait qu'on explique aisément en examinant les mécanismes de reproduction du groupe lui-même. La supériorité, ou plutôt l'excellence de certaines attitudes est aussi un fait social. C'est la constatation d'un certain niveau mesuré selon des standards variables de groupe à groupe, d'époque à époque. Contrairement à ce que Pareto croit, les «capacités » humaines n'existent pas dans la nature à l'état de déterminismes aveugles. Elles sont le produit d'interactions sociales, le résultat d'opérations, d'activités d'inculcation, d'intériorisation, de socialisation de toutes sortes. La «capacité » parétienne est une acquisition sociale, conditionnée par l'origine sociale, par les types plus ou moins différenciés de socialisation; elle s'acquiert et se pratique d'ailleurs selon la condition de classe et la position de classe. Même les capacités les plus valorisées peuvent, d'autre part, ne pas déterminer l'acquisition et l'exercice du pouvoir. D'autres facteurs interviennent, outre la capacité, ou en alternance avec elle, sont susceptibles de permettre l'accès à l'élite. Lesquels ?

Pareto parle parfois du poids de l'origine sociale et de la technique de la corruption comme moyen d'accès ou de maintien dans l'élite d'individus non «capables »; cependant, il croit que la condition normale est la «capacité personnelle».

Les élites peuvent subsister et persister à condition qu'elles se renouvellent continuellement, qu'elles éliminent les éléments dégénérés, qu'elles acceptent en leur sein les éléments nouveaux selon certaines proportions. La circulation - la mobilité - entre la couche inférieure et la couche supérieure doit être surtout verticale, ascendante mais aussi descendante. «Actuellement, dans nos sociétés, l'apport de nouveaux éléments, indispensables à l'élite pour subsister, vient des classes inférieures et principalement des classes rurales. Celles-ci sont le creuset où s'élaborent, dans l'ombre, les futures élites.» (Pareto, 1978: I, 12). Et Pareto ajoute plus loin: "Ce phénomène des nouvelles élites, qui, par un mouvement incessant de circulation, surgissent des couches inférieures de la société, montent dans les couches supérieures, s'y épanouissent et, ensuite, tombent en décadence, sont anéanties, disparaissent, est un des principaux de l'histoire, et il est indispensable d'en tenir compte pour comprendre les grands mouvements sociaux.» (Pareto, 1978: I, 15). «Le mouvement de circulation, qui porte les élites, nées des couches inférieures, au sommet, et qui fait descendre disparaître les élites au pouvoir, est le plus souvent voilé par plusieurs faits. D'abord, comme il est en général assez lent, ce n'est qu'en étudiant l'histoire d'une longue période de temps, de plusieurs siècles, par exemple, qu'on peut percevoir le sens général et les grandes lignes de ce mouvement. L'observateur contemporain, celui qui ne porte ses regards que sur une courte période de temps, n'aperçoit que les circonstances accidentelles. Il voit des rivalités de castes, l'oppression d'un tyran, des soulèvements populaires, des revendications libérales, des aristocraties, des théocraties, des ochlocraties; mais le phénomène al, dont ce ne sont là que des aspects particuliers, lui échappe souvent entièrement.» (Pareto, 1978: I, 34).

Il n'y a donc pas de circulation quand il y a assimilation ou cooptation pure et simple. La circulation se «produit précisément quand des éléments étrangers à l'élite viennent à en faire partie, y apportant leurs opinions, leurs caractères, leurs vertus, leurs préjugés. Mais si, au contraire, ces personnes changent leur manière 
d'être, et d'ennemis deviennent alliés et serviteurs, on a un cas entièrement différent, dans lequel la circulation fait défaut.» (Pareto, 1968: § 2482). En conséquence, circulation est synonyme d'équilibre.

La loi qui gouverne la continuité est la constitution des élites est soumise à une espèce d'anaklasis. "Les aristocraties ne durent pas. Qu'elles qu'en soient les causes, il est incontestable qu'après un certain temps, elles disparaissent. L'histoire est un cimetière d'aristocraties.» (Pareto, 1968: § 2053). «Ce n'est pas seulement quant au nombre que certaines aristocraties sont en décadence: c'est aussi quant à la qualité, en ce sens que l'énergie y diminue, et que se modifient les proportions des résidus qui leur servirent à s'emparer du pouvoir et à le conserver [...]. La classe gouvernante est entretenue, non seulement en nombre, mais ce qui importe davantage, en qualité, par les familles qui viennent des classes inférieures, qui lui apportent l'énergie et les proportions de résidus nécessaires à son maintien au pouvoir. Elle est tenue en bon état par la perte de ses membres les plus déchus.» (Pareto, 1968: § 2054).

Les élites peuvent disparaître pour diverses raisons, qu'on peut réduire à trois : la destruction biologique; le changement des attitudes psychologiques; la décadence.

La destruction biologique frappe les aristocraties d'origine militaire, qui subissent des décimations notables sur les champs de bataille. Le changement des attitudes psychologiques est dû à l'affaiblissement des mobiles culturels, qui met les élites à la merci d'autres mobiles. Parfois cette élite, en proie au désespoir, fait recours à la violence; parfois, par contre, elle supporte, elle se plie comme une brindille au vent. Dans les deux cas, elle est incapable de réaliser des programmes, d'élaborer des projets d'avenir. La décadence dérive du fait que les rôles et les statuts des individus dépendent largement de l'origine sociale. Malheureusement, rien n'assure que les fils soient aussi capables que les pères et qu'il y ait une harmonie entre les dons personnels et les positions sociales. Par conséquent, il est possible que dans les élites se trouvent des individus incapables, qui déchaînent le processus de décadence.

Dans ces conditions comment maintenir la stabilité et la continuité sociales? Il n'y a que deux moyens, selon Pareto, moyens qui peuvent être utilisés simultanément ou alternativement: en éliminant ceux qui contestent et ceux qui risquent de mettre en danger l'ordre social et l'existence de l'élite, et/ou en absorbant les éléments de la classe gouvernée qui peuvent être utiles ou utilisables. Ce processus d'endosmose, par lequel des éléments de la classe gouvernée viennent faire partie de l'aristocratie du pouvoir, est appelé « le phénomène de la circulation sociale». Il faut absolument qu'à l'intérieur d'un système social, il y ait cette circulation. L'élite capable est celle qui montre de l'habileté et de la capacité d'invention pour se renouveler et se rajeunir continuellement. Il peut arriver que la contre-élite se serve, pour éliminer les adversaires au pouvoir, du mécontentement des classes gouvernées ou aussi de l'intervention étrangère. La classe au pouvoir doit alors se défendre. Quels moyens employer? Pareto parle de ruse et de force, et aussi d'un certain consensus passif de la part de la classe gouvernée. Par ruse, il faut entendre la connaissance, la diplomatie, la stratégie, l'utilisation correcte de tous les biens symboliques; par force, il faut entendre non la violence légale exclusivement, mais aussi et davantage la force d'âme, la dévotion à la communauté, le culte de l'idéal, l'esprit de sacrifice. La ruse est classifiée parmi les 
résidus de l'instinct des combinaisons tandis que la force parmi ceux de la persistance des agrégats.

\section{TYPES DE SYSTÈMES SOCIAUX}

Un ordre social «ouvert», une société non «bloquée», est le produit d'un équilibre entre le résidu de l'instinct des combinaisons et le résidu de la persistance des agrégats; entre l'innovation, la découverte et l'invention d'une part, la conformité aux normes, aux valeurs, aux éthos sociaux, aux idéaux traditionnels d'autre part. Les régimes sont caractérisés avant tout par la psychologie des élites. Les différences historiques qui caractérisent les régimes n'ont pas de poids face à ce trait essentiel de l'ordre social.

En bref, la distribution des résidus parmi les individus et parmi les classes sociales est à l'origine des types de systèmes sociaux: un type où prévaut un instinct fort des combinaisons, avec un nombre élevé de spéculateurs, d'entrepreneurs, de réformateurs, d'inventeurs et d'ambitieux capables des entreprises les plus dangereuses, et un type où l'on rencontre une forte concentration du résidu de la persistance des agrégats, avec une prédominance de rentiers, d'individus pour lesquels le passé est un bien présent et futur et qui veulent que rien ne change.

Les spéculateurs prévalent d'habitude plus facilement par la tromperie, par la ruse et par d'autres manipulations, mais ils n'arrivent jamais à maintenir un contrôle prolongé de la situation. Ils sont évincés par les rentiers qui à leur tour sont chassés du pouvoir par les spéculateurs. C'est le mouvement perpétuel, cyclique.

Pareto croit qu'avec l'augmentation de la sécurité individuelle et collective, avec l'accroissement du bien-être, avec la persistance de la coexistence entre les peuples, l'esprit d'entreprise s'éteint, la hardiesse militaire s'affaiblit et les gouvernements sont toujours plus réticents à employer la force. Parallèlement, dans la classe gouvernée, se sont infiltrées et s'y sont établies des valeurs et des normes qui suffisent à faire chanceler les modèles culturels traditionnels. L'autorité est ébranlée; la rébellion devient possible. L'enthousiasme religieux avec lequel la nouvelle situation est vécue par les masses, combinée au déchaînement de nouvelles cupidités, tend à s'imposer par la force. L'ancien équilibre social est remplacé par un nouvel équilibre, en substituant par la force une classe à une autre. La circulation sociale d'un côté retarde la catastrophe en appelant de nouveaux éléments à renforcer l'équipe de la classe gouvernementale, mais de l'autre côté elle hâte cette catastrophe par la désertion d'autres dirigeants qui se mettent à la tête du mouvement révolutionnaire.

Séparation inévitable entre gouvernements et gouvernés? Précarité de tous les mécanismes qui voudraient s'interposer dans cette séparation? Inéluctabilité de la dégénérescence de toutes les classes dirigeantes? La réponse de Pareto est nette. La vie sociale et politique est cyclique. Le changement social est inévitable, mais il s'agit d'un changement d'un type particulier, de surface. Il est en effet dû à une alternance sans fin de minorités, qui ne visent, au delà de tout, qu'à commander. Changement de minorités, donc changement de forme non point changement de la structure du pouvoir, donc changement de substance. Une seule réalité est éternelle: la vie politique et sociale a une stratification élémentaire et fondamentale, 
celle des dominants et des dominés. Elle est oligarchique par essence et tous les moyens sont valables pour que l'élite puisse réaliser ses buts. Ni la démocratie, ni le socialisme, ni le libéralisme, ni le conservatisme ne peuvent réaliser ce qu'ils promettent. Les politiciens promettent toujours un changement total et radical. Leur action peut être utile, mais elle n'est pas en rapport avec la fin qu'ils se proposent. Aussitôt qu'ils ont conquis le pouvoir, ils créent une société qui n'a rien à voir avec celle qu'ils promettaient. La vie sociale est pleine d'embûches, de mensonges, de simulations, d'antagonismes, d'avanies, de cruautés. Les principes éthiques fondamentaux ne sont ni vrais ni faux car ils n'affirment ni démentent rien. Il s'agit de préceptes qui expriment des «out-propositions» tirés de «inpropositions » indémontrables. Leurs force et validité sont garanties uniquement par des argumentations persuasives.

Sans une distribution relativement contrôlée du pouvoir aucun système social ne peut exister ou fonctionner et pourtant l'exercice de ce pouvoir ne peut être éclairé par la science car les actions et les interactions sociales impliquent toujours des mobiles et des choix non-logiques dans la détermination des fins visées.

\section{UNE THÉORIE GÉNÉRALE DU SOCIAL?}

Peut-on dire que Pareto nous a légué une théorie générale fondée sur les événements symptomatiques de la société de son temps et que cette théorie, au croisement de la sociologie, de la psychologie et de l'histoire, est plus proche de l'«histoire immédiate» que de la sociologie standard et qu'elle est aussi une sociologie de la décadence? (Valade, 1990: 299) Que Pareto ait «exploré diverses constellations historiques » sur la base du postulat de l'existence de «constantes dans la nature humaine», c'est indubitable. Cependant il n'a jamais écrit que la «rupture de l'équilibre social par une élite montante au détriment de l'élite en place » est la cause de la décadence «absolue» des sociétés. Il a plutôt affirmé que les ruptures ne sont jamais identiques, qu'elles ne réapparaissent jamais de la même manière et qu'elles dépendent à chaque fois de contextes historiques nouveaux (Freund, 1984: 160-175).

Pareto s'intéresse à l'identité de la nature humaine à travers le temps, à ce qui est constant chez les hommes dans les sociétés, à ce qui est permanent au-delà de la diversité des cultures. Les actions sociales sont déterminées par les passions, les intérêts, les croyances, les préjugés, les illusions et par l'action conjointe de facteurs génétiques et culturels. Ces actions sont composites. En effet, les activités particulières ne s'assemblent jamais en unité et restent des expressions ou des manifestations de constellations historiques spécifiques. Les acteurs sociaux s'influencent réciproquement grâce à des discours persuasifs ou dissuasifs, à l'art de dire ou de taire, de simuler ou de déguiser. L'agir social est toujours plus que toutes les activités particulières réunies. Les systèmes des fins ultimes ne peuvent être saisis par la seule analyse des actions individuelles ou de généralisations présumant l'existence des mécanismes connecteurs des divers éléments. Pour cette raison il faut modéliser, de la façon la plus élémentaire, les phénomènes sociaux, il faut fonder les modélisations sur l'analyse comparative des comportements socioculturels, sur la mise en évidence des prédispositions naturelles qui se manifestent dans les universaux culturels, les croyances communes et les compor- 
tements collectifs, sur l'analyse logique des représentations que l'on donne des interactions. Cette modélisation est relative et provisoire, elle dépend de l'état de nos connaissances et de nos savoirs, du fait que le caractère complexe des faits sociaux dépend de la façon dont on les regarde à un moment donné. Une théorie générale unifiée et absolue n'arrivera jamais à expliquer les conditions d'existence de la société car il est impossible d'en saisir à la fois les réseaux de significations et le système signifiant, d'appréhender les situations dans lesquelles les délibérations, les discussions, les décisions et les choix se vérifient, la manière selon laquelle ils sont structurés en fonction d'une perspective donnée, de même que les réglages effectués en cours de construction et de transformations ainsi que les opérations de détermination, de constitution, de prédication, de mise en relation, d'acceptabilité et de cohésion.

Convaincu que la réalité sociale est produite et reproduite à partir de points de vue et de raisons très diverses, que les théories engendrent les faits et valident ensuite ces mêmes faits qu'elles ont préalablement créés, qu'il est difficile de faire appel à la preuve par calcul lorsqu'il s'agit de faits sociaux, Pareto a tenté de modéliser une théorie des modalités susceptible d'expliquer, sans recourir aux catégories téléologiques et à la causalité, comment, dans la pratique quotidienne, l'on passe de l'intention à l'action.

$\mathrm{Au}$ point de départ des ses analyses, Pareto place les représentations, verbales et non verbales, collectives et individuelles, la raison délibérative entretenue par la «logique» argumentative, opposée à la logique démonstrative et aux systèmes axiomatiques formalisés dépourvus à la fois de sujets et de critères pour l'interprétation. Ensuite il met en évidence les modalités constitutives, non déductives et non formelles, de cette pensée non-logique et puis il essaye d'expliciter «les uniformités que présentent les phénomènes, c'est-à-dire leurs lois, sans avoir en vue aucune utilité pratique directe, sans se préoccuper en aucune manière de donner des recettes ou des préceptes, sans rechercher même le bonheur, l'utilité ou le bien être de l'humanité ou d'une de ses parties. Le but est dans ce cas exclusivement scientifique; on veut connaître, savoir, sans plus.» (Pareto, 1966: 2-3; Pareto, 1968: § 87). Inéluctable, en conséquence, est le rejet des «systèmes absolus », lesquels, selon la formulation d'Alexis de Tocqueville, «font dépendre tous les événements de l'histoire de grandes causes premières se liant les unes aux autres par une chaîne fatale, et qui suppriment, pour ainsi dire, les hommes de l'histoire du genre humain». Pareto, en effet, dédaigne les «systèmes absolus », les «sublimes théories», les «grands paradigmes» (le marxisme, le positivisme, le fonctionnalisme, le durkheimisme et tant d'autres -ismes), il prétend à élaborer des modèles de l'action humaine, une théorie sociale, multiple, inventive, relative, axée sur l'étude de phénomènes circonscrits, contextualisés, une théorie apte à rendre compte du faisceau des déterminations qui aménagent les actions des sujets. Sans renoncer à la rationalité épistémique, il insiste sur le rôle limité du principe de rationalité dans les actions sociales et sur le caractère partial et partiel de leurs descriptions et interprétations par les acteurs sociaux. Les modèles théoriques qu'il propose sont des constructions abstraites, simplifiées, approximatives, de la réalité sociale, ce sont des modèles permettant de déduire les caractéristiques apparentes des comportements collectifs et des phénomènes sociaux. Ces derniers ne sont pas l'effet d'une cause unique, unilinéaire, déterminante, mais d'interactions multiples se déroulant selon des mouvements cycliques. Ces 
interactions et les comportements qu'elles instituent sont non-logiques, non rationnels, mais rationalisés, justifiés avec des arguments apparemment rationnels. En partant des rationalisations des conduites individuelles et des comportements collectifs (dénommés les dérivations et les dérivés), Pareto remonte aux facteurs dissimulés (les résidus) qui les inspirent, les fondent, et qui en rendent envisageable une intelligibilité relative.

Etant donné que la science sociale ne peut pas s'appuyer sur les conduites observables, commensurables, calculables, que l'écart entre l'algèbre de Boole et la pensée naturelle fait que la formalisation est impuissante à saisir le sens et les significations, que les indéterminations, les ambiguïtés et les polyvalences de l'agir social sont indéfinies, Pareto tente de remonter des rationalisations discursives de l'action à des principes primordiaux les rendant possibles. Ces principes (les résidus), dont la psychologie et la biologie peuvent en spécifier la nature, sont identifiés de façon régressive grâce aux analyses des dérivations et des dérivées. Cette option méthodologique, qu'on peut ranger du côté de l'empirisme logique ou de la pragmatique sociale du discours, privilégie les habitudes langagières, les raisonnements, les représentations, la logique brute de la subjectivité irréductible au calcul; elle différencie le langage naturel ou vulgaire propre aux croyances représentées, aux décisions, aux choix, aux actions de la vie quotidienne, du langage artificiel de la science. La logique de l'action est différente de la cognition scientifique. Il n'y a pas un ordre social autonome. Pour cette raison les faits sociaux ne se trouvent inscrits ni dans le monde qui nous entoure ni même dans notre monde intérieur, pour cette raison il est impossible d'enregistrer l'existence de ces faits. Même si l'on admettait qu'il existe quelque part des faits sociaux tout donnés, ils ne suffiraient guère à supporter les déductions. Tous les faits sociaux sont des représentations produites par des opérations d'organisation, de liaison et de complexification, par la généralisation d'actes quotidiens.

L'ordre social n'est pas assuré par des systèmes normatifs mais par des radicaux aux préfixes et suffixes multiples et constituant des totalités signifiantes en rapport avec l'action. Si la société est l'ensemble des ensembles des rapports sociaux, si les rapports sociaux modifient à la fois la nature et les comportements; si la société est l'entrecroisement de séries de générations pesant sur les suivantes par l'intermédiaire de signes, de valeurs et de règles, c'est-à-dire du langage, des échanges et des normes collectives logiques et prélogiques, la science sociale doit éclairer comment les combinaisons entre les classes et les genres de ces archétypes, de ces radicaux dénommés « résidus », donnent lieu à des types de sociétés, analysables et compréhensibles avec une théorie postulant l'existence d'une élite gouvernante et d'une majorité gouvernée. Le système social, sa forme de gouvernement, l'idéologie les rationalisant, les justifiant et les faisant valoir en légitimité, sont expliqués par la prévalence des types socio-psychologiques des spéculateurs et des rentiers. Dans le régime démocratique prédominent les «spéculateurs », «des hommes qui se préoccupent simplement de leurs affaires, et chez lesquels les résidus de la Ie classe sont puissants.» (Pareto, 1968: § 2254) Ils acceptent les nouveautés, les aventures économiques dangereuses, «Aucun échec ne les décourage. [...] Par leur insistance tenace et leur art subtil des combinaisons (Ie classe des résidus) ils surmontent tous les obstacles. Leurs opinions sont toujours celles qui leur sont le plus profitables sur le moment» (Pareto, 1968: $\S 2313)$. 
Pour la sociologie standard l'action rationnelle est celle de l'acteur social qui organise son agir de façon raisonnable et plausible par rapport à un ou à des systèmes de significations irréductibles à une utilité empirique contingente. Les fins sont des données repérables. Pour Pareto les fins sont celles qui se réalisent au point d'équilibre, donc elles sont irréductibles à la rationalité formelle des langages scientifiques. Ainsi, la rationalité de la science s'oppose à celle de l'action et de la décision, la connaissance objective des savants à celle subjective des acteurs, les explications par la raison consciente, ou susceptibles de le devenir, à celles se trouvant à la base de l'agir individuel et social.

La tendance des hommes à argumenter pour rendre plausibles et acceptables leurs comportements et leurs croyances, cette tendance est le fondement de l'existence sociale puisqu'elle réalise l'intersubjectivité des interprétations et garantit les ententes tacites à la base des échanges sociaux. Les discours des acteurs sociaux sont conçus comme les bases mêmes de la socialité. Par l'entremise du langage «on parvient jusqu'aux sentiments des hommes » et, par celui-ci « on peut en conséquence modifier leur activité » (Pareto, 1968: § 1403).

Pareto a voulu construire des modèles et de typologies des processus macrosociologiques à partir de la dichotomie universalisme/particularisme, où l'universalisme est le code et le particularisme le contexte. Il n'élabore pas une théorie de l'équilibre général de la société mais un modèle pour rendre compte des types de systèmes empiriquement déterminés. Et puisqu'il présume l'impossibilité pour la sociologie d'indiquer le moyen approprié à une fin, sa conclusion inévitable est que la rationalité de l'action demeure inconciliable avec la rationalité épistémique, qu'entre les «bonnes raisons» de l'action et la logique de la science la séparation reste absolue. Par conséquent, cette sociologie souligne la différence radicale entre les logiques de l'action sociale et la logique de la science, entre les logiques argumentatives et les logiques démonstratives, l'impossibilité de concilier la pratique avec la théorie, l'action individuelle et sociale et la connaissance scientifique.

Les modèles explicatifs parétiens visent, en dernier lieu, à rendre compréhensibles la thèse selon laquelle la vie sociale est gouvernée par des facteurs prélogiques et non-logiques et selon laquelle ces facteurs sont argumentés et rendus plausibles et recevables postérieurement aux accomplissements des actions.

\section{EPILOGUE}

Si la vie sociale est la résultante de facteurs primordiaux consolidés au cours de l'évolution de l'espèce humaine, si la force et la ruse sont à la base de la vie en société, si la cruauté est éternelle, si l'on est à la merci des illusions et des mythes, si les hommes sont davantage des êtres de foi que de raison, s'ils croient avant de comprendre ou s'ils croient sans comprendre, si le non-logique est à la base des «manières d'agir », alors à quoi bon la connaissance sociologique, à qui s'adresset-elle, à quoi peut-elle servir? Si l'œuvre de Pareto n'exhibe que les illusions et les arguments fallacieux dont les acteurs sociaux enrobent la vérité et l'objectivité, il est alors justifié de juger sa contribution à la connaissance de la vie en société dérisoire et même de donner à cette oeuvre une place infime dans l'histoire de la sociologie. Toutefois il ne faut négliger que Pareto reste convaincu que «sa» 
science sociale produit de la transparence et de l'intelligibilité dans les relations et les interactions sociales mais il est aussi conscient qu'elle n'éclairera jamais les angoisses et les doutes des acteurs sociaux ni servira à faire des choix convenables.

Pour Pareto comprendre pourquoi et comment l'homme croit, agit et produit l'organisation sociale, contrôle ou maîtrise ou subit les conditions de vie en société, sa destinée existentielle individuelle et celle de son groupe d'appartenance, cela appartient au domaine de la vérité scientifique et non à celui de l'utilité sociale. Cette compréhension peut être vraie mais néfaste socialement. La vérité et l'utilité ne concordent jamais dans la société. Pareto est convaincu que la sociologie n'est pas une sorte de médecine pour traiter les organisations sociales malades, les relations de groupe, les problèmes sociaux, voire la société «en crise». Elle n'est pas non plus la conseillère du Prince, l'assistante sociale de la «bonne société ». Pareto est opposé à toute forme de pragmatisme gestionnaire, d'interventionnisme pour rationaliser les rapports sociaux. Pour lui la sociologie est une aventure, strictement contrôlée, de la pensée, c'est une approche intellectuelle charnière entre la philosophie et l'histoire, entre la psychologie et l'économie. Il s'oppose à la transformation de la sociologie en une activité assemblant, rapprochant et composant les «faits », en une pratique, parmi tant d'autres, nécessaire à la gestion efficiente des sociétés bureaucratisées. Il veut maintenir vivante, à côté de la pensée qui calcule, propre aux sciences instrumentales, une pensée qui médite et réfléchit, une pensée capable de Gelassenheit.

Pareto nous rappelle infatigablement que la sociologie doit travailler de concert avec toutes les sciences de l'homme et de la société car le présent est le produit du passé. C'est la permanence du passé à donner sa configuration au présent, à en faire ce qu'il est actuellement. Cette permanence du passé dans le présent est la charpente de notre conscience, de notre identité sociale, des institutions, des valeurs, des normes; elle est l'échalas des structures sociales actuelles. Les hommes créent un monde d'objets, de significations, de contraintes dans la durée. Ils en assurent la stabilisation et la permanence grâce aux institutions. Celles-ci deviennent peu à peu des sujets qui transforment leurs créateurs en objets. Nous créons les règles, les valeurs, les signes; nous agissons au travers d'eux. Ils condensent et parfois cristallisent les expériences sociales. Le recours quasi automatique à ces expériences facilite l'action individuelle et collective, ordonne la vie sociale, facilite les interactions, fixe les champs du possible, préserve les énergies et stimule les motivations et les orientations.

Ce monde construit se place, au cours des années, devant nous comme un monde indépendant de nous, implacable dans sa propre autonomie et dont la destinée peut parfois différer de celles des sujets individuels. D'où la duplicité de l'action sociale: elle est choix et contrainte à la fois mais dans le temps. Cela signifie que l'action dépend des situations historiques auxquelles le sujet est confronté. Notre condition historique nous donne le cadre de nos expériences, façonne nos conduites, facilite certains comportements. Dès lors la biologie et la psychologie, la sociologie et l'histoire sont les faces d'une même médaille. Elles s'occupent des mêmes réalités et rendent compte des mêmes processus, c'est-àdire des pourquoi et des comment les générations des hommes vivent ensembles, produisent des significations, donnent du sens à des choses qui autrement n'en auraient point, créent des mécanismes pour légitimer et faire valoir des choix arbi- 
traires, pour les perpétuer, les changer, les échanger, pour donner un ordre là où il n'y avait qu'insignifiance et indifférenciation, inconsistance et contingence.

Pareto s'inscrit en faux contre la croyance que la société contemporaine est une société radicalement nouvelle, que la modernité produit un progrès indéfini, que la science peut résoudre tous les problèmes, que les sociétés humaines peuvent être façonnées selon des projets volontaristes. Il est de l'avis que la nature humaine change lentement. C'est elle à constituer le fondement de tous nos comportements, c'est à elle qu'il faut arriver, par les biais des représentations et des discours des acteurs sociaux, pour donner une première description, approximative, relative, susceptible de nouveaux compléments, de l'agir et du fonctionnement sociaux, pour trancher de la vérité ou de la fausseté d'une assertion empirique, pour établir la validité d'une proposition entrant dans une argumentation.

Pareto affirme qu'il n'y a pas de science unique, que le monde social est préinterprété et défini en tant que tel par les activités changeantes de ses membres. Pour le saisir, il faut donc présupposer les caractères signifiants de la vie sociale. En d'autres termes, il faut postuler la préexistence d'une capacité symbolique, d'expériences construites dans le langage et dans la pensée, en bref des sujets agissants, raisonnants, faisant appel à des préconstruits implicites, dont on peut faire des typologies mais jamais en établir l'inventaire. Les résidus permettent à Pareto de postuler ces préconstruits, fruits de l'histoire évolutive de l'espèce humaine, à la fois normes de vérité, de raison, de critique. Mais pour appliquer ces normes, pour distinguer les opinions des arguments rationnels, les prémisses absurdes de celles plausibles ou valables, il faut s'appuyer sur les préconstruits culturels, sur les énoncés préthéoriques, sur les principes et les codifications particulières placés à la base des normes pratiques et cognitives.

En rapportant les dérivations aux résidus, Pareto est de l'avis qu'ainsi il sera possible de rendre plus ou moins transparent ce qui est voilé, caché, incompréhensible ou insaisissable dans l'agir social et dans les formes de cet agir mais aussi dans la statique et dans la dynamique de la société. Pour lui la recherche doit aider à comprendre que le présent n'est pas la seule forme de vie possible, l'unique et exclusive réalité, qu'il n'y a pas de solutions scientifiques aux problèmes sociaux, que la science sociale ne doit pas se laisser fourvoyer par le mirage des solutions prétendument scientifiques aux modes d'organisation et de fonctionnement de la société et des relations sociales.

\section{Université de Lausanne}

\section{RÉFÉRENCES BIBLIOGRAPHIQUES}

Aron, R., 2006, Les sociétés modernes. Textes rassemblés et introduits par Serge Paugam, Paris, Quadrige/Puf.

Barbut, M., 2007, Mesures des inégalités. Modélisation mathématique et traitements statistiques, Genève, Droz.

Bobbio, N., 1996, Saggi sulla scienza politica in Italia, Bari, Laterza.

Boudon, R., 1968, A quoi sert la notion de «structure»? Essai sur la signification de la notion de structure dans les sciences humaines, Paris, Gallimard.

- 1971, La crise de la sociologie. Questions d'épistémologie sociologique, Genève, Droz. 
Bourdieu, P., Chamboredon, J.C., Passeron, J.C., 1972, Le métier de sociologue. Préalables épistémologiques, Paris-La Haye, Mouton.

Bourdieu, P., 1972, Esquisse d'une théorie de la pratique, Genève, Droz.

Busino, G., 1974, Gli studi su Vilfredo Pareto oggi. Dall'agiografia alla critica (1923-1973), Roma, Bulzoni.

- 1999, «Lire Pareto aujourd'hui ?» in: A. Bouvier (sous la direction de), Pareto aujourd'hui, Paris, Puf.

- 2006, Sugli studi paretiani all'alba del XXI secolo, «Rivista storica italiana», CXVIII, fasc. 2.

- 2007, Contributi alla storia della cultura economica del '900, s.i.é \& 1 .

Freund, J., 1974, Pareto : la théorie de l'équilibre, Paris, Seghers.

- 1984, La décadence. Histoire sociologique et philosophique d'une catégorie de l'expérience humaine, Paris, Sirey.

Levi-Strauss, C., 1955, Tristes tropiques, Paris, Plon.

Pareto, V., 1964, Cours d'économie politique, Genève, Droz, 1964.

- $\quad 1965$, Ecrits sur la courbe de la répartition de la richesse, Genève, Droz.

- 1966, Manuel d'économie politique, Genève, Droz.

- 1968, Traité de sociologie générale, Genève, Droz.

- $\quad$ 1978, Les systèmes socialistes, Genève, Droz.

- 1987, Marxisme et économie pure, Genève, Droz.

Passeron J.C., 1995, «Weber et Pareto: La rencontre de la rationalité dans l'analyse sociologique » in: L.A. Gerard-Varet, J.-C. Passeron (sous la direction de), Le modèle et l'enquête. Les usages du principe de rationalité dans les science sociales, Paris, Ed. de 1'E.H.E.S.S.

- 2000, L'économie dans la sociologie, dans Economia, Sociologia e Politica nell'opera di Vilfredo Pareto. A cura di C. Malandrino e R. Marchionatti, Firenze, Olschki.

Valade, B., 1990, Pareto. La naissance d'une autre sociologie, Paris, Puf. 